\title{
EVIDENCIAS DE ESTRUCTURA POBLACIONAL Y ESTRUCTURA GENÉTICA ESPACIAL A ESCALA FINA EN POBLACIONES ARgentinas de ACACIA FURCATISPINA (FABACEAE)
}

\author{
EVIDENCES OF POPULATION STRUCTURE AND SPATIAL GENETIC STRUCTURE \\ AT fine SCALE IN ARgentinean POPULATIONS OF ACACIA FURCATISPINA
}

(FABACEAE)

\author{
Elias N. Cerdeira ${ }^{1}$, Beatriz O. Saidman ${ }^{1}$ (D) y Carolina L. Pometti ${ }^{1 *}$ (D)
}

\begin{abstract}
1. Laboratorio de Genética, Departamento de Ecología, Genética y Evolución, Instituto IEGEBA (CONICET- UBA), Facultad de Ciencias Exactas y Naturales, Universidad de Buenos Aires, Intendente Güiraldes s/n, Pabellón 2, Ciudad Universitaria (1428), Buenos Aires, Argentina

*cpometti@ege.fcen.uba.ar; caritoege@gmail.com
\end{abstract}

\section{Citar este artículo}

CERDEIRA, E. N., B. O. SAIDMAN \& C. L. POMETTI. 2019. Evidencias de estructura poblacional y estructura genética espacial a escala fina en poblaciones argentinas de Acacia furcatispina (Fabaceae). Bol. Soc. Argent. Bot. 54: 79-91.

DOI: http://dx.doi. org/10.31055/1851.2372.v54. n1.23587

Recibido: 7 Septiembre 2018 Aceptado: 21 Diciembre 2018 Publicado: 28 Marzo 2019 Editora: Viviana Solís Neffa (D)

ISSN versión impresa 0373-580X ISSN versión on-line 1851-2372

\section{SUMMARY}

Background and aims: Acacia furcatispina belongs to the subgenus Aculeiferum. Currently, there are no studies in population genetics in this species. The aim of this work was to investigate the genetic diversity, population structure and spatial genetic structure at fine scale (SGS) in Argentinean sample sites by means of molecular markers.

Materials and Metods: Two sample sites of $A$. furcatispina were studied with the AFLP technique. Three primer combinations were selected for this study.

Results: The three primer combinations revealed a total of 121 AFLP bands. The percentage of polymorphic loci showed a mean value of $86.8 \%$ and the mean heterozygocity was 0.33 . The Bayesian analysis performed with software STRUCTURE detected three clusters $(K=3)$, corresponding one to Pasaje Pozo Zuni (PP) sample site and two for Cerro de la Gloria (CG) sample site, showing a subdivision of this last one (CG1 and CG2). The AMOVA showed that the great component of variability was represented within populations. The SGS detected significant structuration at short and middle distances in PP.

Conclusions: Based on the results of the present work, we recommend to sample trees far apart $10 \mathrm{~m}$ to minimize the kinship between individuals. These results present the first study in genetic diversity, population structure and spatial genetic structure at fine scale in $A$. furcatispina and all the analyses made here result of interest for management and conservation of this species.

\section{KEY WORDS}

Acacia, AFLP, CDA, genetic structure, SGS, STRUCTURE.

\section{RESUMEN}

Introduccion y objetivos: Acacia furcatispina, pertenece al subgénero Aculeiferum. Actualmente no hay estudios sobre la diversidad y estructura genética de esta especie. El objetivo de este trabajo fue estudiar la diversidad genética, estructura poblacional, y la estructura genética espacial a escala fina (SGS) en localidades argentinas de esta especie.

Materiales y Métodos: Se estudiaron dos localidades de la especie A. furcatispina mediante la técnica de AFLP. Se seleccionaron 3 combinaciones de cebadores.

Resultados: Con las combinaciones de cebadores seleccionadas, se revelaron un total de 121 bandas. El porcentaje de loci polimórficos presentó un valor medio de $86,8 \%$ y la heterocigosis 0,33 . El análisis bayesiano STRUCTURE mostró que los individuos analizados pertenecen a tres grupos genéticos $(K=3)$, uno correspondiente a la localidad Pasaje Pozo Zuni (PP) y dos, a Cerro de la Gloria (CG), subdividida en dos poblaciones (CG1 y CG2). El AMOVA indicó que la mayor parte de la variabilidad se encuentra contenida dentro de las poblaciones. El análisis SGS detectó estructuración local significativa a cortas y medianas distancias en la población PP.

Conclusiones: Basado en los resultados del presente trabajo, se recomienda muestrear árboles a más de 10 metros de distancia para minimizar el parentesco entre individuos. Estos resultados proveen el primer estudio acerca de la diversidad, estructura genética y estructura a escala espacial fina de Acacia furcatispina y todos los análisis realizados resultan de interés para el manejo y conservación de esta especie.

\section{Palabras clave}

Acacia, AFLP, CDA, diversidad genética, SGS, STRUCTURE. 


\section{INTRODUCCIÓN}

El género Acacia pertenece a la familia Fabaceae, subfamilia Mimosoideae. Fue establecido por Miller en 1754, basándose en el nombre propuesto por Tournefort en 1694. Es un género de distribución pantropical, que habita regiones tropicales y subtropicales de América, Asia, África y Australia, siendo Australia el continente donde alcanza su mayor diversidad (Cialdella, 1984).

Actualmente, la delimitación del género Acacia es controversial dado que algunos autores lo consideran como varios géneros agrupados (Orchard \& Maslin, 2005; Smith et al., 2006; van Rijckevorse, 2006; Moore et al., 2011). A pesar de esto, en este trabajo consideraremos el género Acacia s.l.

Se estima que aproximadamente 1450 especies integran el género en la actualidad. Para Argentina se han citado 19 especies, incluidas en dos subgéneros (Rico-Arce, 2007).

Acacia furcatispina Burkart (A. gilliesi Steud.), en particular, es una especie perteneciente al subgénero Aculeiferum. Se distribuye en Sudamérica: Argentina, Bolivia y Paraguay, donde es conocida vulgarmente como "garabato blanco", "garabato macho", "mochuelo", "teatin", "brea" o "tinticaco", entre otros (Rico-Arce, 2007). Las especies de Acacia, en general, tienen la capacidad de fijar nitrógeno atmosférico, proveen madera para combustibles, extractos medicinales, taninos, gomas, madera de obra, fibras, sombra y alimento para animales silvestres y domésticos (Pometti et al., 2012). Algunos trabajos recomiendan, entre otras especies del género, a $A$. furcatispina para la forestación; además, su madera presenta características consideradas deseables para el aprovechamiento forestal (Bravo et al., 2006). Su regeneración natural es por semilla y su agente de dispersión más importante es el ganado doméstico. La germinación de las semillas se acelera debido al proceso de escarificación que reciben al pasar por el intestino del ganado (bovino, ovino y equino) (Abedini et al., 2000).

La distribución geográfica de esta especie dentro de la República Argentina alcanza las provincias del centro y noroeste, citándose Buenos Aires, Catamarca, Córdoba, La Rioja, Mendoza, Salta, San Juan, San Luis, Santiago del Estero y Tucumán (Cialdella, 1984).
Es importante conocer los factores que modifican la variabilidad genética debido a la fragilidad de las especies y ecosistemas, ya que pueden ser afectadas por las alteraciones del medio ambiente. Actualmente la presión sobre los recursos naturales ha llevado a importantes pérdidas de diversidad, especialmente en lo que respecta a especies forestales (Bessega et al., 2017). Para entender cómo ha evolucionado la estructura genética poblacional es necesario saber qué procesos y fuerzas evolutivas han sido importantes durante su desarrollo y mantenimiento. En este sentido, la técnica de AFLP resulta útil para el estudio de la genética de poblaciones debido a sus altos niveles de polimorfismo (Vos et al., 1995) y debido a que estos marcadores están menos afectados por la presión de selección (Nybom, 2004).

Hasta el momento, las especies del subgénero Aculeiferum han sido poco estudiadas en Sudamérica desde el punto de vista genético poblacional (Pometti et al., 2013, 2016), por lo que en el presente trabajo se propone estudiar la diversidad, estructura poblacional y estructura genética a escala fina (SGS) de dos localidades argentinas de A. furcatispina provenientes de dos ecorregiones diferentes. Para ello se prevé encontrar que: 1) la mayor parte de la diversidad genética estará representada dentro de las poblaciones ya que esto sucede en la mayoría de las especies de Acacia estudiadas, por ejemplo A. albida Del. (Joly et al., 1992), A. senegal (L.) Willd. (Chiveu et al., 2008; Omondi et al., 2010), A. caven (Mol.) Mol. (Pometti et al., 2011, 2012), A. curvifructa Burkart y A. farnesiana (L.) Willd. (Pometti et al., 2015), A. visco Lorentz ex Griseb (Pometti et al., 2016), A. aroma Gillies ex Hook (Pometti et al., 2018); y 2) dado que la dispersión de A. furcatispina es mediante el ganado doméstico, como fue mencionado previamente, y que esta especie comparte los mismos sistemas de dispersión de polen y fruto que otras especies del género, como por ejemplo, $A$. aroma (Pometti et al., 2018), se espera encontrar estructura genética espacial a escala fina.

\section{Materiales y Métodos}

\section{Área de estudio y colecciones}

Se analizaron dos localidades de la especie $A$. furcatispina, provenientes de dos ecorregiones 


\section{E. N. Cerdeira et al. - Estudio de parámetros genético poblacionales en Acacia furcatispina}

diferentes. Una perteneciente a la provincia de Mendoza, de la localidad Cerro de la Gloria (CG: -32,885; -68,892), ecorregión Monte de llanuras y mesetas (Burkart, et al. 1999). Esta ecorregión se caracteriza por presentar un clima templado-árido y escasas precipitaciones, las temperaturas medias anuales son del orden de 10 a $14^{\circ} \mathrm{C}$, las amplitudes térmicas son marcadas, los suelos presentan características salinas y pedregosas $\mathrm{y}$, en cuanto a su vegetación, predomina el jarillal (Burkart et al., 1999). La otra población proviene de la provincia de Santiago del Estero, de la localidad Pasaje Pozo Zuni (PP: -27,965933; -63,9242), ecorregión Chaco seco. Esta ecorregión se caracteriza por presentar clima cálido subtropical, con zonas que presentan las máximas temperaturas del continente, la temperatura media anual varía de norte a sur desde $23^{\circ} \mathrm{C}$ hasta cerca de $18^{\circ} \mathrm{C}$, las precipitaciones varían entre 500 y $700 \mathrm{~mm}$ anuales, son marcadamente estivales y el tipo de vegetación característica es el bosque xerófilo (Fig. 1A-C) (Burkart et al., 1999). Se muestrearon 20 árboles adultos por localidad y de cada árbol se coleccionaron hojas que se mantuvieron en bolsas herméticas con sílica gel hasta la extracción del ADN. Los ejemplares de referencia fueron depositados en el herbario del Instituto de Botánica Darwinion (SI) (Ejemplares CG 893, Colector C. Bessega y PP 912, Colector M. Ewens).

\section{Extracción de ADN}

Se utilizó como material experimental hojas de árboles adultos y se extrajo ADN genómico total utilizando el kit comercial Quiagen DNeasy Plant (Quiagen, Valencia, CA), siguiendo su respectivo protocolo.

La técnica de AFLP fue realizada siguiendo el protocolo de Vos et al. (1995), con pequeñas modificaciones (Pometti et al., 2016). Se utilizaron tres combinaciones de cebadores selectivos: E-ACA/M-CTT (C1), E-AGG/M-CAG (C3) y E-AAC/M-CAA (C4). Los productos de la amplificación selectiva fueron visualizados en geles de poliacrilamida teñidos con nitrato de plata. El tamaño de las bandas AFLP fue de 90 a 400 pb.

\section{Diversidad genética}

Cada banda AFLP fue considerada como un locus simple, bialélico, con un alelo amplificable (dominante) y otro nulo (recesivo). Las bandas
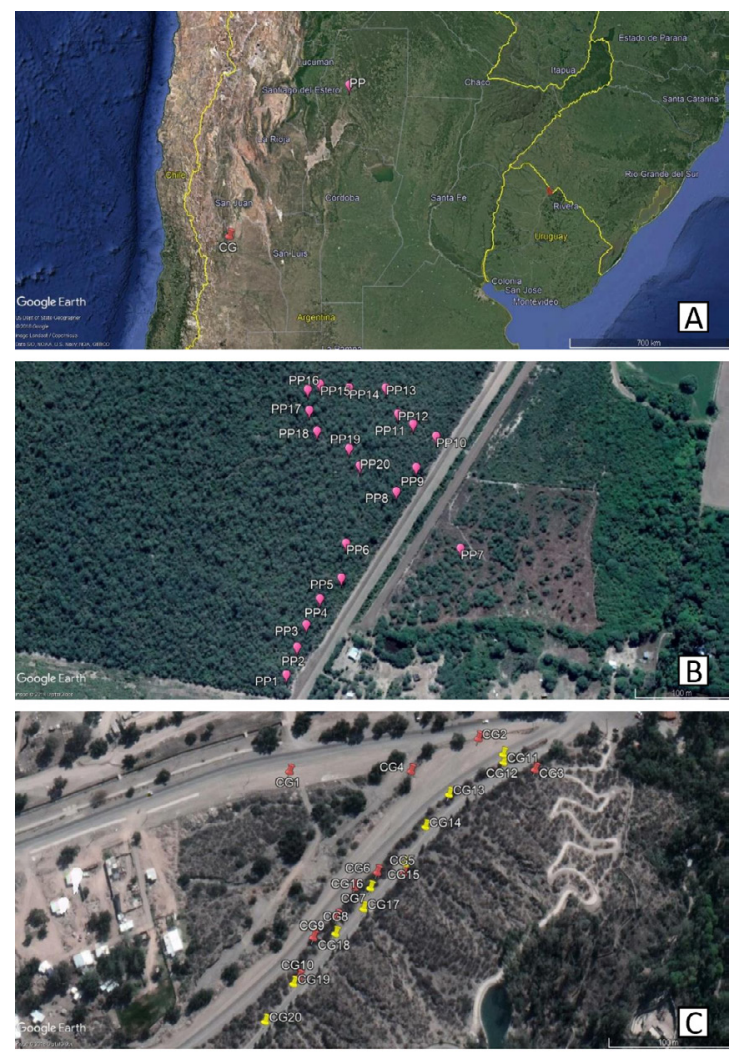

Fig. 1. Localización de las localidades de Acacia furcatispina estudiadas. A: Localización de ambas localidades en el mapa de la República Argentina. B: Disposición espacial de los individuos en la localidad Pasaje Pozo Zuni (PP), provincia de Santiago del Estero. C: Disposición espacial de los individuos de la localidad Cerro de la Gloria (CG), provincia de Mendoza. Se indican del 1 al 20 los individuos de cada localidad.

con la misma distancia de migración fueron consideradas homólogas. Se construyó una matriz de presencia (1) o ausencia (0) de bandas AFLP.

El método bayesiano de verosimilitud implementado en el programa BAYESCAN v2.1 (Foll \& Gaggiotti, 2008) fue utilizado para identificar la existencia de variantes con divergencia no neutral entre las 2 poblaciones $\left(F_{S T}\right.$-outliers $)$. Se utilizó un período de burn-in de 10000, un intervalo de thinning de 10, el número de iteraciones fue de 25000. El estudio de diversidad genética y estructura poblacional, por consiguiente, se llevó a cabo con los loci neutros. 
Las frecuencias alélicas fueron estimadas utilizando el método bayesiano con distribución previa no uniforme, según fue descrito por Zhivotovsky (1999), implementado en el programa AFLPsurv (Vekemans, 2002) siguiendo el enfoque de Lynch \& Milligan (1994).

El porcentaje de loci polimórficos, la heterocigosidad y el estimador $F_{S T}$ no jerárquico (Wright, 1978) también se estimaron con el mismo programa.

La capacidad informativa de las combinaciones de cebadores AFLP se estimó a través de los siguientes parámetros. El contenido de información polimórfica (PIC, polymorphic information content) fue calculado de acuerdo a lo propuesto por Roldan-Ruiz et al. (2000).

$$
\text { PIC }=1-\sum_{i=1}^{k} P_{i}^{2}
$$

Donde $k$ es el número total de alelos detectados para un locus y $P_{i}$ es la frecuencia de $i$-ésimo alelo en el conjunto de genotipos analizado. El valor máximo de PIC para marcadores dominantes es de 0,5.

El índice del marcador (MI, marker index) determinado mediante la metodología propuesta por Varshney et al. (2007) fue calculado como producto de dos funciones: el PIC y la proporción múltiple efectiva (EMR, effective multiplex ratio). El EMR de un cebador se define como (Milbourne et al., 1997).

$$
M I=P I C * E M R
$$

Donde $n$ es el número de loci polimórficos y $\beta$ es la fracción de loci polimórficos.

El poder resolutivo ( $R P$, resolvent power) de cada combinación de cebadores fue estimado de acuerdo a lo propuesto por Prevost \& Wilkinson (1999). El valor de una posición de banda particular se puede estimar simplemente como su similitud con la condición óptima (50\% de los genotipos conteniendo esa banda). La capacidad informativa de una banda $\left(I_{b}\right)$ varía entre 0 y 1 y se calcula como:

$$
I_{b}=1-(2 *|0.5-p|)
$$

Donde $p$ es la proporción de genotipos que contienen el locus. Si todas las bandas son óptimamente informativas, el cebador más útil será aquel que genere la mayor cantidad de posiciones de banda. La capacidad de un cebador para distinguir entre genotipos se representa como la suma de la información aportada por cada loci.

$$
R P=\sum I_{b}
$$

La utilidad de un marcador es un balance entre el nivel de polimorfismo detectado y la extensión a la cual un ensayo puede identificar múltiples polimorfismos. El índice del marcador $(M I)$ provee una estima acertada de la utilidad del marcador.

\section{Estructura genética poblacional}

Para analizar la estructura poblacional de las localidades de $A$. furcatispina, analizadas en este trabajo, se implementó el programa STRUCTURE v.2.3.4 (Pritchard et al., 2009). Los parámetros de corrida utilizados fueron: período de burn-in de 10000 repeticiones y 25000 iteraciones de MCMC (Cadenas de Markov Monte Carlo). Se seleccionó el modelo no admixture con frecuencias alélicas independientes. Se ensayó con valores de $K$ dentro del rango de 1 a 5 . Los valores de $K$ fueron promediados a partir de 10 iteraciones. Se seleccionó el $K$ óptimo aplicando el criterio de Pritchard (2000). Los datos fueron procesados utilizando el programa STRUCTURE HARVESTER (Earl \& von Holdt, 2012) para obtener el promedio de las 10 iteraciones. Los resultados del STRUCTURE fueron editados con el programa CLUMP 1.1.2 (Jakobsson \& Rosenberg, 2007) y Distruct 1.1 (Rosenberg, 2004) para obtener la representación gráfica.

Otro método para estudiar la estructura genética poblacional utilizado fue el AMOVA con los niveles jerárquicos poblaciones/individuos (empleando los grupos genéticas revelados por STRUCTURE). Este análisis fue realizado con el programa Geno v.0.1 (Dyer, 2008).

\section{Análisis discriminante canónico}

Para conocer cuáles son las variables de clasificación (presencia/ausencia de banda) que mejor diferencian las poblaciones genéticas inferidas mediante el análisis STRUCTURE se realizó un análisis discriminante canónico con el programa STATISTICA 7.0 (StatSoft, Inc. 2004).

\section{Análisis de la estructura espacial a escala fina}

Para analizar la estructura genética espacial a escala fina (SGS), se utilizó la aproximación de Hardy (2003), estudiando cada población a través de su coeficiente de parentesco $\left(F_{i j}\right)$. El coeficiente de endogamia asumido fue de $F_{I S}=0$ (valor predeterminado por el programa). Para establecer las relaciones entre las clases de distancia geográficas y las similitudes genéticas fue estimada la pendiente de la regresión 


\section{E. N. Cerdeira et al. - Estudio de parámetros genético poblacionales en Acacia furcatispina}

de los coeficientes de parentesco transformados en logaritmos de las clases de distancia $\left(b_{F}\right)$. Para determinar la significancia estadística de $F_{1}$ (el coeficiente de parentesco medio entre individuos pertenecientes a la primera clase de distancia) y el $b_{F}$, se utilizaron los límites superior e inferior del intervalo de confianza del $95 \%$ de $F_{i j}$, los cuales fueron definidos a partir de 10000 permutaciones de los individuos dentro de las poblaciones. El estadístico $S p$ se estimó para cada población basado en la pendiente de la reoresión de los coeficientes de parentesco como $S p={ }^{-b_{F}} /\left(1-F_{1}\right)$. El estadístico $S p$ resume la intensidad de la SGS permitiendo comparar cuantitativamente este parámetro entre especies y/o poblaciones (Vekemans \& Hardy, 2004). Todas las estimaciones de SGS fueron realizadas con el programa SPAGeDi v1.5 (Hardy \& Vekemans, 2002).

Una estimación indirecta del flujo génico a partir de las estimas de SGS puede ser realizada asumiendo que se ha alcanzado el equilibrio de aislamiento por distancia en la estructura genética a escala fina. En dichos casos, el flujo génico puede ser expresado en términos del tamaño del vecindario $\left(N_{b}\right)$ como $N_{b} \cong 4 \pi D_{E} \sigma_{g}{ }^{2}$, donde $D_{E}$ es la densidad efectiva de la población y $\sigma_{g}$ es la distancia media padre-hijo al cuadrado y puede ser estimada como la inversa de $S p$ siendo $N_{b}=\left(F_{1}-1\right) / b_{F}$. En este trabajo sólo estimamos el tamaño del vecindario $\left(N_{b}\right)$ y la dispersión $\left(\sigma_{g}\right)$, en los casos en que se detectó SGS significativo, usando la densidad censada de las poblaciones y tres valores efectivos preestablecidos $\left(1 / 2,1 / 4\right.$ y $1 / 10$ de la $\left.D_{E}\right)$.

\section{Resultados}

\section{Diversidad genética}

Con las tres combinaciones de cebadores utilizadas en la técnica de AFLP se obtuvieron 121 loci. Cuando se realizó la búsqueda de posibles $F_{S T}$-outliers mediante el programa BAYESCAN (con un valor umbral de $q$ del $10 \%$ ) no se detectaron loci bajo selección.

El porcentaje de loci polimórficos $(P L P)$ entre cebadores varió desde 78,9\% a $100 \%$ (Tabla 1 ), mientras que entre poblaciones varió desde 86,0 $\%$ a $88,4 \%$ (Tabla 2 ). La heterocigosis esperada $\left(H_{E}\right)$ varió desde 0,30 en Cerro de la Gloria 2 (CG2) hasta 0,37 en Cerro de la Gloria 1 (CG1) (Tabla 2).

Además se detectaron bandas únicas en las tres poblaciones. Para PP, se encontraron 2 y 9 bandas únicas para los primers $\mathrm{C} 3 \mathrm{y} \mathrm{C} 4$, respectivamente. Para CG1, se encontraron 3 bandas únicas para el primer $\mathrm{C} 1$. Para CG2 se encontró 1 banda única para el primer $\mathrm{C} 3$.

Tabla 1. Polimorfismo y capacidad informativa de las tres combinaciones seleccionadas de cebadores AFLP analizados en poblaciones de Acacia furcatispina provenientes de dos localidades. Porcentaje de loci polimórficos $(P L P)$, contenido de información polimórfica $(P I C)$, índice del marcador $(M I)$ y poder resolutivo $(R P)$

\begin{tabular}{|ccccccccc|}
\hline $\begin{array}{c}\text { Combinación } \\
\text { de cebadores }\end{array}$ & Bandas total & $\begin{array}{c}\text { Bandas } \\
\text { monomórficas }\end{array}$ & $\begin{array}{c}\text { Bandas } \\
\text { polimórficas }\end{array}$ & PLP & PIC & MI & RP \\
\hline C1 & 41 & 0 & 41 & 100 & 0,26 & 10,66 & 22,45 \\
C3 & 38 & 8 & 30 & 78,9 & 0,29 & 8,7 & 16,8 \\
C4 & 42 & 4 & 38 & 90,5 & 0,27 & 10,26 & 23 \\
Total & 121 & 12 & 109 & & 0,82 & 29,62 & 62,25 \\
Promedio & 40 & 4 & 36 & 89,8 & 0,27 & 9,87 & 20,75 \\
\hline
\end{tabular}

Tabla 2. Resumen de la diversidad genética basada en el estudio de 121 loci AFLP analizados en 3 poblaciones de Acacia furcatispina. Porcentaje de loci polimórficos (PLP), heterocigosis esperada $(H)$, error estándar (SE $(H))$, porcentaje de la varianza debido al muestreo de individuos y de loci (Varl\% y VarL \%, respectivamente).

\begin{tabular}{|cccccc|}
\hline Población & PLP & H & SE (H) & Varl\% & VarL\% \\
\hline CG1 & 88,4 & 0,37 & 0,01 & 44,5 & 55,5 \\
CG2 & 86,0 & 0,30 & 0,01 & 40,9 & 59,1 \\
PP & 86,0 & 0,32 & 0,01 & 22,5 & 77,5 \\
\hline
\end{tabular}


Al analizar la capacidad informativa de los cebadores se observa que $\mathrm{C} 1$ y $\mathrm{C} 4$ presentan valores de $M I$ y $R P$ más altos $(\mathrm{C} 1, R P=22,45$ y $M I=10,66 ; C 4, R P=23,00$ y $M I=10,26)$ que los de $\mathrm{C} 3(R P=16,80$ y $M I=8,70)$, mientras que los valores de $P I C$ son similares para las 3 combinaciones de cebadores, oscilando entre 0,26 y 0,29 (Tabla 1 ).

El porcentaje de varianza debido al muestreo de individuos (VarI $=35,97 \%$ en promedio) es menor al porcentaje de la varianza debido al muestreo de loci ( $\operatorname{VarL}=64,03 \%$ en promedio) (Tabla 2).

\section{Estructura genética poblacional}

El análisis de los datos mediante el programa STRUCTURE reveló la presencia de tres grupos $(K=3)$. En dicho valor se alcanza el plateau para el mayor valor medio de la densidad de la probabilidades $(\ln P(D)=-2081,56)$ sugiriendo que el número óptimo de $K$ es 3 . En este análisis, los individuos de la localidad PP formaron un único grupo genético, mientras que los individuos de $\mathrm{CG}$ se dividieron en dos grupos diferenciados (CG1 y CG2). El grupo formado por los individuos de PP, presenta mayor cantidad de individuos de origen genético diferente (Fig. 2A). Esto se evidencia cuando $K=2$ donde todos los individuos pertenecientes a PP forman una misma población junto con los individuos de CG2 (Fig. 2B)

El valor del $F_{S T}$ no jerárquico, obtenido mediante el programa AFLPsurv, fue de 0,12 $(P<0,001)$.

Los resultados del AMOVA arrojaron que la variabilidad genética entre poblaciones representó el $24,4 \%$ de la varianza total, mientras que el $75,6 \%$ de la varianza total ocurrió dentro de las poblaciones. La estima de $\Phi_{S T}(0,24)$ resultó significativa, lo que evidenciaría la existencia de estructura poblacional (Tabla 3).

\section{Análisis discriminante canónico}

El análisis discriminante realizado con los tres grupos genéticos inferidos por el análisis STRUCTURE, en el cual la localidad de CG se dividió en dos grupos de 10 individuos cada uno (CG1 y CG2) muestra que los dos primeros ejes canónicos representan el 94,8 \% y el 5,2\% de la varianza molecular (Fig. 3) y que 10 de los 121 loci analizados fueron significativos para diferenciar a los individuos de las respectivas
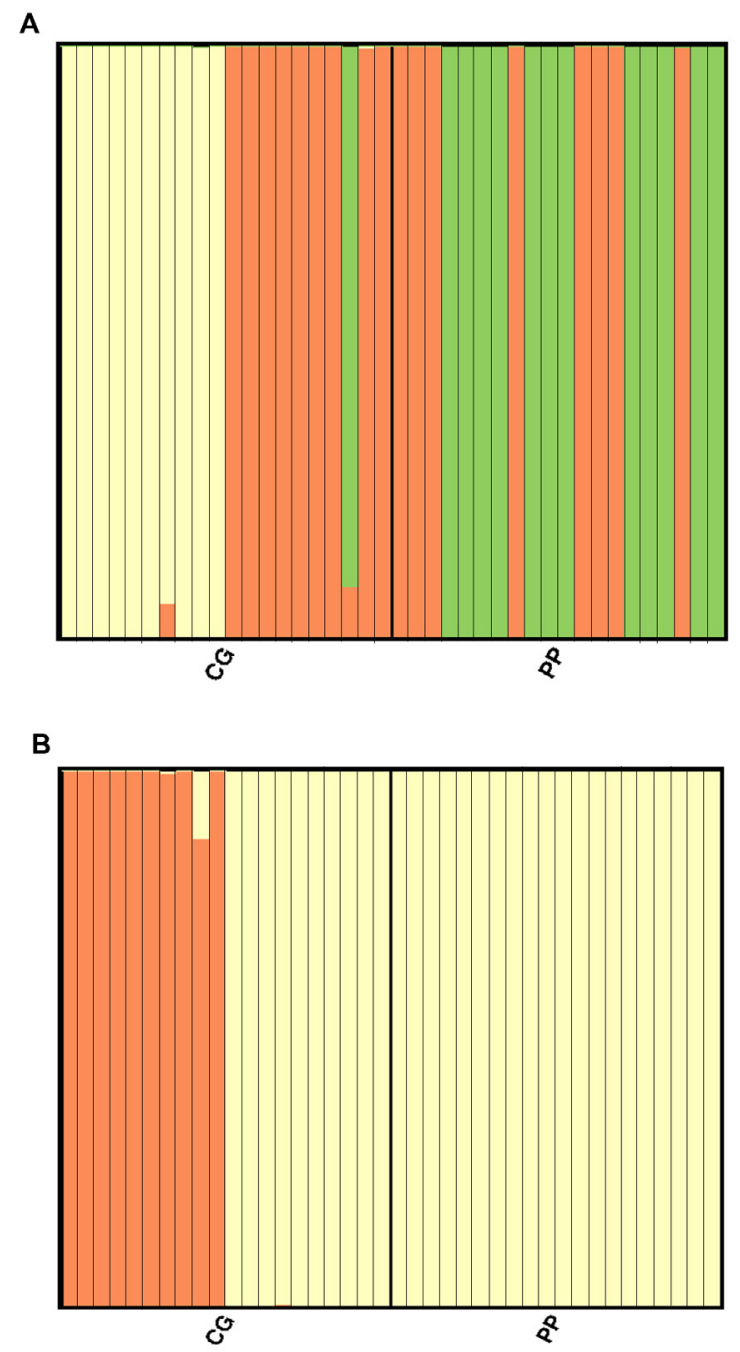

Fig. 2. Agrupamiento de individuos producido por el programa STRUCTURE. A: Para un valor óptimo de $K=3$. B: Para un valor de $K=2$. Cada individuo se representa por una barra vertical coloreada. El mismo color en diferentes individuos indica que pertenecen al mismo grupo.

poblaciones (Tabla 4). Se observa que de los 10 loci seleccionados, 6 pertenecen al cebador $\mathrm{C} 1,2$ al cebador $\mathrm{C} 3$ y otros 2 , a C4 (Tabla 4).

La correcta clasificación de los individuos basada en los patrones de AFLP fue del $100 \%$ para las tres poblaciones (Tabla 5).

La representación gráfica de dichos ejes muestra una clara diferenciación de los grupos prestablecidos de Acacia furcatispina. 
E. N. Cerdeira et al. - Estudio de parámetros genético poblacionales en Acacia furcatispina

Tabla 3. Estructura poblacional estimada mediante un análisis de la varianza molecular (AMOVA) en 3 poblaciones de Acacia furcatispina en base al estudio de 121 loci AFLP. Grados de libertad (GL). Suma de las desviaciones al cuadrado $(\mathrm{SSD})^{*} \mathrm{P}<0,05$.

\begin{tabular}{|ccccccc|}
\hline Origen & GL & SSD & $\begin{array}{c}\text { Componente de } \\
\text { la varianza }\end{array}$ & \% Varianza & $\boldsymbol{\varphi}$ & P \\
\hline Entre poblaciones & 2 & 85,79 & 2,75 & 24,4 & 0,24 & 0,001 \\
Dentro de poblaciones & 37 & 315,4 & 8,52 & 75,6 & - & - \\
\hline Total & 39 & 401,19 & 11,27 & 100 & - \\
\hline
\end{tabular}

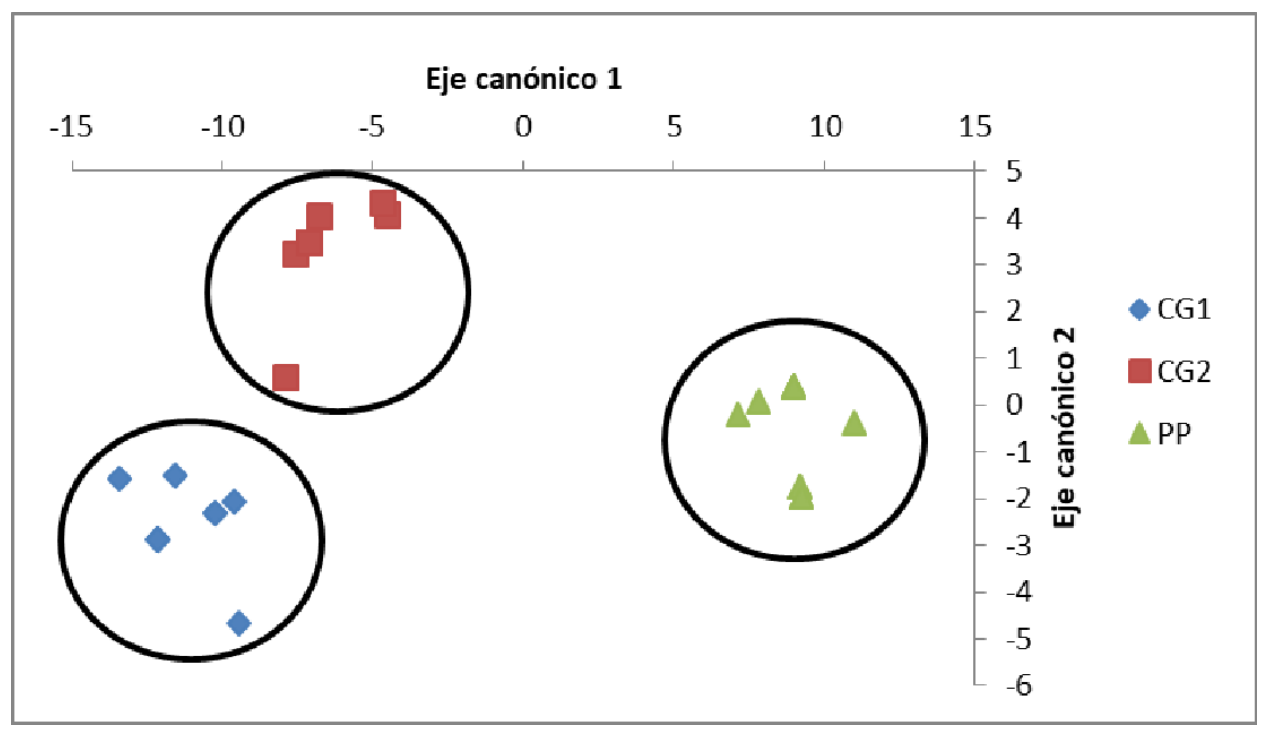

Fig. 3. Representación gráfica de los dos ejes canónicos de los 3 grupos de poblaciones de Acacia furcatispina a partir de los datos de 121 loci AFLP.

Tabla 4. Análisis stepwise a partir de la presencia/ausencia de 121 bandas AFLP en tres poblaciones de Acacia furcatispina. Se muestran los 10 loci que fueron significativos para diferenciar a los individuos a nivel poblacional.

\begin{tabular}{|cccccccc|}
\hline Locus & Test & Valor & F & Efecto DF & Error DF & P \\
C1_4 & Wilks & 0,233 & 46,150 & 2 & 28 & 0,00 \\
C1_5 & Wilks & 0,715 & 5,579 & 2 & 28 & 0,01 \\
C1_14 & Wilks & 0,584 & 9,975 & 2 & 28 & 0,00 \\
C1_21 & Wilks & 0,779 & 3,975 & 2 & 28 & 0,03 \\
C1_34 & Wilks & 0,224 & 48,474 & 2 & 28 & 0,00 \\
C1_35 & Wilks & 0,137 & 88,107 & 2 & 28 & 0,00 \\
C3_3 & Wilks & 0,055 & 239,967 & 2 & 28 & 0,00 \\
C3_35 & Wilks & 0,530 & 12,404 & 2 & 28 & 0,00 \\
C4_3 & Wilks & 0,544 & 11,714 & 2 & 28 & 0,00 \\
C4_7 & Wilks & 0,376 & 23,276 & 2 & 28 & 0,00 \\
\hline
\end{tabular}


Tabla 5. Clasificación de los individuos de las 3 poblaciones de Acacia furcatispina usando el análisis discriminante basado en 121 loci AFLP.

\begin{tabular}{|ccccc|}
\hline Población & CG1 & CG2 & PP & $\begin{array}{c}\text { Clasificación } \\
\text { correcta (\%) }\end{array}$ \\
\hline CG1 & 0 & 4 & 6 & 0 \\
CG2 & 0 & 6 & 4 & 60 \\
PP & 0 & 2 & 18 & 90 \\
Total & 0 & 12 & 28 & 60 \\
\hline
\end{tabular}
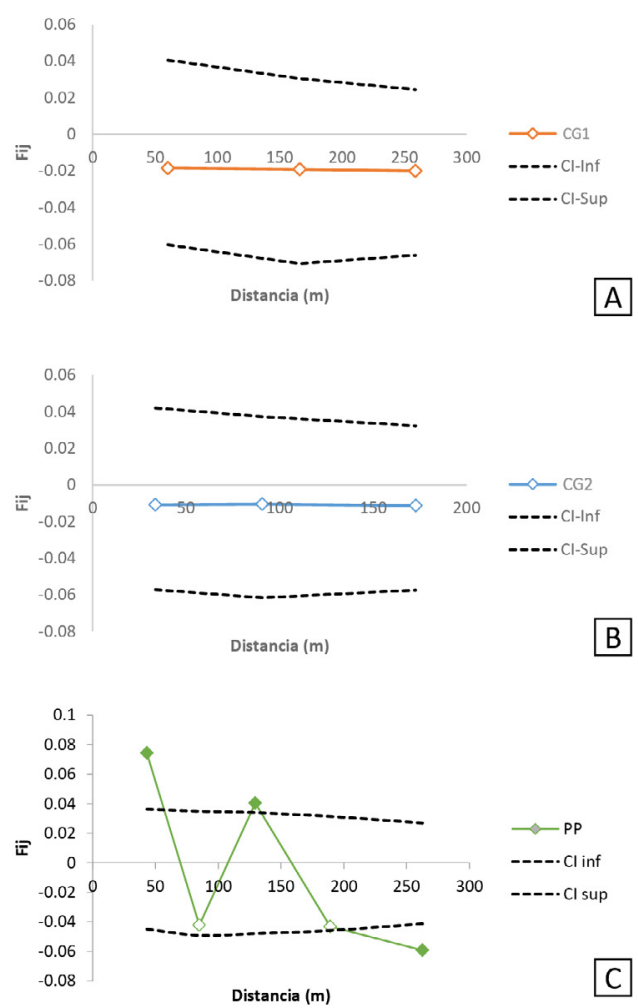

Fig. 4. Correlogramas que representan la estructura espacial a escala fina de las tres poblaciones de Acacia furcatispina provenientes de las localidades de Cerro de la Gloria (A y B) y Pasaje Pozo Zuni (C). Los rombos rellenos indican valores de $F_{i j}$ significativos. La línea punteada representa los intervalos de confianza del 95\% (10000 permutaciones).
Análisis de la estructura espacial a escala fina

El análisis de estructura espacial a escala fina de las tres poblaciones (Fig. 4A-C) evidenció estructuración local significativa para cortas y medianas distancias sólo en la población PP (a 43,2 y $129,5 \mathrm{~m}$ ) (Fig. 4A-C).

El tamaño del vecindario se calculó para la población que presentó SGS significativo (PP) y fue de aproximadamente 15 individuos. La estimación de la distancia de dispersión de polen y semillas se realizó considerando cuatro densidades efectivas diferentes, obteniéndose valores que oscilan entre 2 y 6,5 metros (Tabla 6).

\section{Discusión}

Dada la importancia ecológica y económica de la especie Acacia furcatispina en Argentina, en este estudio, se analizó la diversidad genética, estructura poblacional y estructura genética espacial a escala fina (SGS) de la especie en dos localidades pertenecientes a dos ecorregiones diferentes.

Las 15 bandas únicas de AFLP identificadas en este trabajo proveen la base para identificar individuos provenientes de las tres poblaciones para programas de manejo forestal, silvicultura, conservación.

La capacidad informativa de las combinaciones de cebadores AFLP reveladas por el PIC y el $M I$ han sido ampliamente utilizadas en muchos estudios de diversidad genética (Varshney et al., 2007; Shen et al., 2010; Pavithra et al., 2014; Khadivi-Khub et al., 2015). Prevost \& Wilkinson (1999) propusieron un nuevo concepto para analizar el poder discriminativo de los cebadores cuantificado por medio del coeficiente $R P$. El $M I$ fue usado para estudiar cuán útil es cada combinación de cebadores. El máximo $M I(10,66)$ se encontró para la combinación $\mathrm{C} 1$ (M-CAA/E-AAC), este índice fue similar para la combinación C4 (M-CTT/E-ACA) $(10,26)$, pero

Tabla 6. Estimación de la estructura genética espacial a escala fina (SGS) en tres poblaciones de Acacia furcatispina. Sp es la cuantificación de la SGS, $F_{1}$ es el coeficiente de parentesco multilocus entre individuos para la primer clase de distancia, $b_{F}$ es la regresión de la pendiente del logaritmo natural de la primer clase de distancia del coeficiente de parentesco $(F), N_{b}$ es el tamaño del vecindario, $D_{E}$ es la

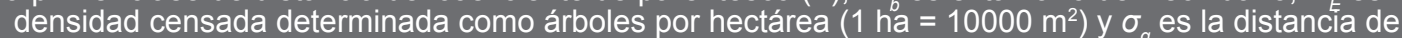
dispersión génica (expresada en metros) usando cuatro densidades efectivas estimadas. ${ }^{*} \mathrm{P}<0,01$.

\begin{tabular}{|cccccccccc|}
\hline & $\mathbf{S p}$ & $\mathbf{F}_{1}$ & $\mathbf{b}_{F}$ & $\mathbf{N}_{\mathbf{b}}$ & $\mathbf{D}_{\mathrm{E}}$ & $\boldsymbol{\sigma}_{\mathrm{g}}\left(\mathbf{D}_{\mathrm{E}}\right)$ & $\boldsymbol{\sigma}_{\mathrm{g}}\left(\mathbf{D}_{\mathrm{E}} / \mathbf{2}\right)$ & $\boldsymbol{\sigma}_{\mathrm{g}}\left(\mathbf{D}_{\mathrm{E}} / \mathbf{4}\right)$ & $\boldsymbol{\sigma}_{\mathrm{g}}\left(\mathbf{D}_{\mathrm{E}} / \mathbf{1 0}\right)$ \\
\hline $\mathbf{C G 1}$ & 0,001 & $-0,000$ & $-0,001$ & - & 0,11 & - & - & - & - \\
$\mathbf{C G 2}$ & 0,029 & 0,030 & $-0,028$ & - & 0,12 & - & - & - & - \\
$\mathbf{P P}$ & 0,068 & 0,074 & $-0,063$ & 14,75 & 0,15 & 2,1 & 2,9 & 4,2 & 6,6 \\
\hline
\end{tabular}




\section{E. N. Cerdeira et al. - Estudio de parámetros genético poblacionales en Acacia furcatispina}

más bajo para la combinación C3 (M-CAA/E-AAG) $(8,7)$. Los valores de $P I C$ fueron similares para las tres combinaciones de cebadores utilizadas en este trabajo. Estos valores fueron similares a los obtenidos en $A$. caven $(P I C=0,3)$ (Pometti et al., 2015) y en Satureja bachtiarica Bunge $(P I C=0,41)$ (KhadiviKhub et al., 2015). El $R P$ mostró el valor más alto para las combinaciones C4 (M-CTT/E-ACA) y C1 (M-CAA/E-AAC). Estos valores, cuando fueron comparados con otras especies de Acacia, resultaron mayores que los registrados para $A$. farnesiana $(\mathrm{RP}=$ $15,5)$ y $A$. curvifructa $(\mathrm{RP}=12,4)$, pero menores que los de $A$. caven $(\mathrm{RP}=35,1)$. En resumen los valores de $P I C, M I$ y $R P$ estimados para las tres combinaciones de cebadores pueden ser considerados relativamente altos, indicando que pueden ser efectivamente usados para discriminar individuos de diferentes poblaciones.

La diversidad genética observada en A. furcatispina es relativamente alta $(H=0,33)$ cuando es comparada con otras especies que comparten las mismas características de historia de vida (Nybom, 2004) y es similar a los valores observados en otras especies del género como $A$. curvifructa $(H=0,21)$ (Pometti et al., 2015), A. visco $(H=0,20)$ (Pometti et al., 2016), A. senegal $(H=0,26)$ (Chiveu et al., 2008) y $A$. aroma $(H=0,20)$ (Pometti et al., 2018) que fueron analizadas también con marcadores dominantes.

La estructura poblacional fue estudiada por medio del programa STRUCTURE, el cual mostró que el número óptimo de grupos genéticos $(K)$ es 3. La localidad de Cerro de la Gloria (CG), Mendoza, fue dividida en dos poblaciones genéticas, mientras que la localidad de Pasaje Pozo Zuni (PP), Santiago del Estero, se mantuvo como un único grupo genético (población). Sin embargo, cuando $K=2$ los individuos pertenecientes a PP y CG2 se agrupan en un mismo grupo genético evidenciando un posible ancestro común para estos dos grupos. Esto se confirma cuando $K=3$, ya que en PP se pueden observar individuos con orígenes genéticos diferentes (provenientes de CG2) mientras que en CG1 y CG2 no hay mezcla en el origen genético. Una posible explicación para la división observada en la localidad de Cerro de la Gloria podría deberse a que la misma fue cosechada en dos años distintos por dos grupos de colección diferentes. Los individuos pertenecientes al primer grupo fueron muestreados en el año 2016, mientras que los individuos del segundo grupo fueron muestreados en el año 2017. Ambos grupos coexisten en simpatría. Una posible hipótesis podría ser que los individuos del primer y segundo grupo de CG pudieran ser de edades diferentes y esto revela una heterogeneidad en el agrupamiento según STRUCTURE. Si ambos grupos fueran de edades diferentes, podrían haber estado sometidos a diferentes condiciones ambientales, actividades antropogénicas, podas, ramoneos, entre otros, y esto afectaría el criterio de selección de los individuos. Otra posible explicación es que los individuos de las dos poblaciones formen parte de distintos eventos de reforestación con poblaciones de orígenes diferentes. Esto sería factible debido a que los individuos muestreados se encuentran en el área metropolitana de la ciudad de Mendoza y a los costados de una ruta. Además, esta localidad es un área muy deforestada como se puede observar en el mapa y una posibilidad es que no todos los individuos de Cerro de la Gloria sean provenientes de poblaciones naturales, sino que sean producto de la reforestación. Todo lo antes dicho podría estar reflejado en el agrupamiento observado con el análisis del STRUCTURE. En un intento de corroborar esta hipótesis se realizaron mediciones morfológicas sobre el material de herbario de ambas poblaciones, pero no se encontraron diferencias significativas que justifiquen este agrupamiento (los datos no se presentan en este trabajo). En el caso de la población de Pasaje Pozo Zuni (PP) se observa un alto grado de heterogeneidad en el origen genético de los individuos. Esto podría deberse al flujo génico de esta población con poblaciones vecinas de la misma especie que no fueron estudiadas en este trabajo. Esta localidad se encuentra densamente forestada por esta y otras especies de Acacia y otros géneros, lo que favorece un mayor intercambio genético entre los individuos muestreados.

Mediante el AMOVA se observó que la mayor parte de la variabilidad genética se encuentra contenida dentro de las poblaciones $(75,6 \%)$. En la literatura se ha comprobado que las especies longevas y de fecundación cruzada tienden a mantener la mayor parte de su variabilidad dentro de las poblaciones (Hamrick \& Godt, 1989). También se encontraron altos niveles de variabilidad genética dentro de las poblaciones al realizar un AMOVA en otras especies sudamericanas y africanas de Acacia: $91 \%$ en poblaciones de A. senegal var kerensis (Omondi et al., 2010), $86 \%$ en poblaciones of $A$. senegal analizadas como un todo (Chiveu et al., 2008), 68,5 $\%$ en poblaciones de $A$. caven (Pometti et al., 2012) y $80 \%$ en poblaciones de $A$. visco (Pometti et al., 
2013). El análisis de la varianza molecular también mostró evidencias de estructura poblacional con un valor de $\Phi_{S T}=0,24(\mathrm{P}=0,001)$. El análisis mediante el $F_{S T}$ no jerárquico, mostró un valor bajo $(0,12)$, pero altamente significativo $(P<0,001)$ también evidenciando la existencia de estructura genética. De acuerdo a la revisión de los índices de estructura poblacional para marcadores dominantes realizada por Nybom (2004) ambos coeficientes deberían coincidir cuando se calculan sobre el mismo conjunto de datos. En este trabajo, ambos coeficientes son del mismo orden de magnitud y altamente significativos, pero no coinciden exactamente. Ejemplos reportados en la literatura muestran que no se han encontrado evidencias de estructura poblacional por medio del $F_{S T}$ de Wright en Acacia senegal y A. albida (Joly et al., 1992; Omondi et al., 2010) mientras que el AMOVA sí resultó significativo. Esto puede deberse a la forma de calcular ambos índices. El $\Phi_{S T}$ es calculado a través de un método fenético que no contempla supuestos evolutivos (solo las varianzas dentro y entre poblaciones) (Dyer, 2008) mientras que el $F_{S T}$ de Wright (1951) se calcula a través de la heterocigosis.

Para respaldar el agrupamiento observado con STRUCTURE, se realizó un análisis discriminante canónico (CDA). Los resultados de estos análisis en conjunto, aunque preliminares, indicarían que la localidad de CG se encuentra dividida en dos poblaciones genéticas.

Los resultados del porcentaje de la varianza debida al muestreo de individuos resultaron más bajos que aquellos debido al muestreo de loci, esto indicaría la robustez de este estudio dado que aquí se estudiaron una mayor cantidad de loci que de individuos y esta sería la situación óptima, acorde a estos resultados.

La fragmentación del hábitat amenaza el mantenimiento de la biodiversidad en muchos ecosistemas. En general, las consecuencias genéticas de la degradación del hábitat se focalizan en la reducción del tamaño poblacional y el aumento del aislamiento espacial de las poblaciones remanentes. No obstante, en algunos casos, la fragmentación podría incrementar el flujo génico entre las poblaciones remanentes, eliminando estructuras genéticas locales (Young et al., 1996). Por tanto, es importante estudiar la estructura genética poblacional cuando se produce degradación del hábitat, con el fin de diseñar planes de manejo para mantener la diversidad genética de las especies.
Estudios preliminares demostraron que la SGS está correlacionada con el sistema de apareamiento, la historia de vida y la densidad poblacional (Vekemans \& Hardy, 2004). Además, el estadístico $S p$ sintetiza la intensidad de la SGS y es útil para comparaciones de la fuerza de la SGS en diferentes poblaciones. En este trabajo, dos de las tres poblaciones mostraron un valor de $S p$ (PP presenta $\mathrm{Sp}=0,068$ y $\mathrm{CG} 1, \mathrm{Sp}=0,029$ ) comparable al obtenido para poblaciones de otra especie de Acacia que presentan valores entre 0,01 y 0,07 (Pometti et al., 2018). Sin embargo, los valores de $S p$ resultaron mayores que los hallados para Schinus molle $(S p=0,021)$ (Lemos et al., 2015) y Prosopis alba $(S p=0,003)$ (Bessega et al., 2016), además, el tamaño del vecindario $(\mathrm{Nb})$ calculado para la población PP resultó similar al de algunas poblaciones de A. aroma (de 15 a 64 individuos) (Pometti et al., 2018). Estas especies comparten los mismos caracteres de historia de vida con $A$. furcatispina. Sin embargo, la dispersión génica $\left(\sigma_{g}\right)$ resultó mucho menor en $A$. furcatispina (de 2 a 7 metros aproximadamente) que en $A$. aroma (De 45 a $864 \mathrm{~m}$ ) (Pometti et al., 2018), aunque comparten los mismos agentes polinizadores y de dispersión de semillas. Esto podría deberse al tamaño de la población analizada. Sin embargo, según Hardy et al. (2006) un tamaño muestral menor a 70 individuos puede ser suficiente cuando el tamaño del vecindario $(\mathrm{Nb})$ resulta menor o igual a $50 \mathrm{y}$ los marcadores son muy informativos, por ejemplo, muchos loci como AFLP y/o varios alelos por locus como los SSR.

\section{Conclusiones}

Dado los altos niveles de deterioro que presentan los ecosistemas de la Argentina es necesario desarrollar planes de manejo que permitan mitigar sus efectos y conservar la diversidad genética de las poblaciones. Para ello, es imprescindible la caracterización mediante marcadores moleculares de las poblaciones de una especie que permitan determinar el número de grupos genéticamente diferentes presentes en cada región (Bessega et al., 2017). El análisis de la SGS provee información que debe ser utilizada para el muestreo de individuos y recolección de semillas para planes de conservación y reforestación ex situ. Por lo tanto, en este estudio se recomienda 


\section{E. N. Cerdeira et al. - Estudio de parámetros genético poblacionales en Acacia furcatispina}

muestrear árboles a más de 10 metros de distancia para minimizar el parentesco entre individuos.

Los resultados en conjunto de este trabajo proveen el primer estudio acerca de la diversidad, estructura genética y estructura genética espacial a escala fina de Acacia furcatispina y todos los análisis realizados resultan de interés para el manejo y conservación de esta especie en Argentina.

\section{CONTRIBUCIÓN DE LOS AUTORES}

Todos los autores participaron en la conceptualización y la escritura del manuscrito. ENC y CLP realizaron el análisis formal, la investigación, y el diseño de la metodología.

\section{Agradecimientos}

Los autores de este trabajo agradecen al Ing. Mauricio Ewens por el material provisto para la cosecha de las muestras. Este trabajo fue financiado por la Agencia Nacional de Promoción Científica y Tecnológica (PICT-2016-0388 a CLP y PICTO 2011 -0081 OTNA a BOS).

\section{Bibliografía}

ABEDINI, W., P. BOERI, L. MARINUCCI, M. RUSCITTI \& L. SCELZO. 2000. Biotécnicas aplicadas a especies forestales nativas. Invest. Agr.: Sist. Recur. For. 9: 31-43.

BESSEGA, C., C. L. POMETTI, M. EWENS, B. O. SAIDMAN \& J. C. VILARDI. 2016. Fine-scale spatial genetic structure analysis in two Argentine populations of Prosopis alba (Mimosoideae) with different levels of ecological disturbance. Eur. J. Forest Res. 135: 495-505. https://doi.org/10.1007/s10342-016-0948-9

BESSEGA, C., C. L. POMETTI, B. O. SAIDMAN \& J. C. VILARDI. 2017. Contribución de estudios genético poblacionales a la conservación de especies nativas de Argentina de interés forestal. CEI 67: 25-35.

BRAVO, S., A. GIMÉNEZ, A., J. MOGLIA. 2006. Caracterización anatómica del leño y evolución del crecimiento en ejemplares de Acacia aroma y Acacia furcatispina en la Región Chaqueña, Argentina. Bosque 27: 146-154.

https://dx.doi.org/10.4067/S0717-92002006000200009
BURKART, R., N. O. BARBARO, R. O. SANCHEZ \& D. A. GOMEZ. 1999. Ecorregiones de la Argentina. Administración de Parques Nacionales, Buenos Aires.

CHIVEU C. J., O. G. DANGASUK, M. E. OMUNYIN \& F. N. WACHIRA. 2008. Genetic diversity in Kenyan population of Acacia senegal (L.) Willd revealed by combined RAPD and ISSR markers. African $J$. Biotechnol. 7: 2333-2340.

CIALDELLA, A. M. 1984. El género Acacia (Leguminosae) en la Argentina. Darwiniana 25: 59-111.

DYER, R. J. 2008. GeneticStudio: Population genetic software that doesn't suck. http://dyerlab.bio.vcu.edu.

EARL, D. A. \& B. M. VON HOLDT. 2012. STRUCTURE HARVESTER: a website and program for visualizing STRUCTURE output and implementing the Evanno method. Conservation Genet. Resour. 4: 359. https://doi.org/10.1007/s12686-011-9548-7.

FOLL, M. \& O. E. GAGGIOTTI. 2008. A genome scan method to identify selected loci appropriate for both dominant and codominante markers: a Bayesian perspective. Genetics 180: 977-993.

HAMRICK, J. L. \& M. J. W. GODT 1989. Allozyme diversity in plant species. En: Brown AHD, Clegg MT, Kahler AL, Weir BS, eds. Plant population genetics, breeding and genetic resources. Sunderland, MA: Sinauer, 43-63.

HARDY, O. J. 2003. Estimation of pairwise relatedness between individuals and characterization of isolation-by-distance processes using dominant genetic markers. Molec. Ecol. 12: 1577- 1588.

HARDY O. J., L. MAGGIA, E. BANDOU, P. BREYNE, H. CARON, M. E. CHEVALLIER, A. DOLIGEZ, C. DUTECH, A. KREMER, C. LATOUCHE-HALLE, V. TROISPOUX, V. VERON \& B. DEGEN. 2006. Fine-scale genetic structure and gene dispersal influences in 10 neotropical tree species. Molec. Ecol. 15: 559-571.

HARDY, O. J. \& X. VEKEMANS. 2002. Spagedi: a versatile computer program to analyse spatial genetic structure at the individual or population levels. Molec. Ecol. Notes. 2: 618-620.

JAKOBSSON, M. \& N. A. ROSENBERG. 2007. CLUMPP: a cluster matching and permutation program for dealing with label switching and multimodality in analysis of population structure. Bioinformatics 23: 1801-1806.

JOLY H. I., M. ZEH-NLO, P. DANTHU, C. AYGALENT. 1992. Population genetics of an African acacia, Acacia albida. I. Genetic diversity of populations from West Africa. Austral. J. Bot. 40: 59-73.

KHADIVI-KHUB, A., H. SALEHI-ARJMAND, K. MOVAHEDI \& J. HADIAN. 2015. Molecular and morphological variability of Satureja bachtiarica in Iran. Pl. Syst. Evol. 301: 77-93. 
LEMOS, R. P. M., C. B. D'OLIVEIRA \& V. M. STEFENON. 2015. Genetic structure and internal gene flow in populations of Schinus molle (Anacardiaceae) in the Brazilian Pampa. Tree Genet. Genomes. 11: 75.

https://doi.org/ 10.1007/s11295-015-0885-7

LYNCH, M. \& B. G. MILLIGAN. 1994. Analysis of population genetic structure with RAPD markers. Molec. Ecol. 3: 91-99.

MILBOURNE, D., R. MEYER, J. E. BRADSHAW, E. BAIRD, N. BONAR, J. PROVAN, W. POWELL \& R. WAUGH. 1997. Comparison of PCR-based marker systems for the analysis of genetic relationships in cultivated potato. Molec. Breed. 3: 127-136.

MOORE, G, G. F. SMITH, E. FIGUEIREDO, S. DEMISSEW, G. LEWIS, B. SCHRIRE, L. RICO \& A. E. VAN WYK. 2010. Acacia, the 2011 Nomenclature Section in Melbourne, and beyond. Taxon 59: 1188-1195.

NYBOM, H. 2004. Comparison of different nuclear DNA markers for estimating intraspecific genetic diversity in plants. Molec. Ecol. 13: 1143-1155.

OMONDI, S. F., E. KIREGER, O. G. DANGASUK, B. CHIKAMAI, D. W. ODEE, S. ORCHARD, A. E. \& B. R. MASLIN. 2005. The case for conserving Acacia with a new type. Taxon 54: 509-512.

PAVITHRA, H. R., M. B. SHIVANNA, K. CHANDRIKA, K. T. PRASANNA \& B. POMETTI, C. L., J. C. VILARDI \& B. O. SAIDMAN. 2011. Mating system parameters and genetic structure in Argentinean populations of Acacia caven (Leguminosae, Mimosoideae). Pl. Syst. Evol. 292: 25-32. https://doi:10.1007/s00606-010-0389-8

POMETTI, C. L., C. F. BESSEGA, J. C. VILARDI \& B. O. SAIDMAN. 2012. Landscape genetic structure of natural populations of Acacia caven in Argentina. Tree Genet. Genomes. 8: 911-924. https:// doi:10.1007/ s11295-012-0479-6

POMETTI, C. L., C. F. BESSEGA, J. C. VILARDI \& B. O. SAIDMAN. 2013. Comparison of mating system parameters and genetic structure in three natural scenarios of Acacia visco (Leguminosae, Mimosoideae). Pl. Syst. Evol. 299: 761-771. https://doi:10.1007/s00606-013-0759-0

POMETTI, C. L., C. F. BESSEGA, J. C. VILARDI, A. M. CIALDELLA \& B. O. SAIDMAN. 2015. Genetic diversity within and among two Argentinean and one Mexican species of Acacia (Fabaceae). Bot. J. Linn. Soc. 177: 593-606. https://doi:10.1111/boj.12262

POMETTI, C. L., C. F. BESSEGA, J. C. VILARDI, M. EWENS \& B. O. SAIDMAN. 2016. Genetic variation in natural populations of Acacia visco (Fabaceae) belonging to two sub-regions of Argentina using AFLP. Pl. Syst. Evol. 302: 901-910. https://doi.org/10.1007/s00606-016-1306-6.
POMETTI, C. L., C. F. BESSEGA, A. M. CIALDELLA, M. EWENS, B. O. SAIDMAN \& J. C. VILARDI. 2018. Spatial genetic structure within populations and management implications of the South American species Acacia aroma (Fabaceae). PLOS ONE 13: e0192107.

https://doi.oirg/10.1371/journal.pone.0192107.

PREVOST, A. \& M. J. WILKINSON. 1999. A new system of comparing PCR primers applied to ISSR fingerprinting of potato cultivars. Theor. Appl. Genet. 98: 107-112.

PRITCHARD, J. K., M. STEPHENS \& P. DONNELLY. 2000. Inference of population structure using multilocus genotype data. Genetics 155: 945-959.

PRITCHARD, J. K., X. WEN \& D. FALUSH. 2009. STRUCTURE ver. 2.3.4. University of Chicago, Chicago, USA. Disponible en: http://pritch. bsd. uchicago.edu/.

RICO-ARCE, M. L. 2007. American species of Acacia (Leguminosae, Mimosoideae). Comisión Nacional para el conocimiento y uso de la Biodiversidad (CONABIO). México, D.F.

ROLDAN-RUIZ, I., J. DENDAUW, E. VANBOCKSTAELE, A. DEPICKER \& $\mathrm{M}$. DELOOSE. 2000. AFLP markers reveal high polymorphic rates in ryegrasses (Lolium spp.). Molec. Breed. 6: 125-134.

ROSENBERG, N. A. 2004. Distruct: a program for the graphical display of population structure. Molec. Ecol. Notes. 4: 137-138.

SHEN, J. L., X. N. JIA, H. Q. NI, P. G. SUN, S. H. NIU \& X. Y. CHEN. 2010. AFLP analysis of genetic diversity of Jatropha curcas grown in Hainan, China. Trees 24: 455-462.

SMITH, G. F., A. E. VAN WYK, M. LUCKOW \& B. SCHRIRE. 2006. Conserving Acacia Mill. With a conserved type. What happened in Vienna? Taxon 55: 223-225.

STATSOFT, Inc. 2004. STATISTICA for Windows 7.0 (Computer Program Manual). StatSoft, Inc., Tulsa.

VAN RIJCKVORSE, P. 2006. Acacia: What did happen at Vienna? Anales Jard. Bot. Madrid. 63: 107-110.

VARSHNEY, R. K., K. CHABANE, P. S. HENDRE, R. K. AGGARWAL \& A. GRANER. 2007. Comparative assessment of EST-SSR, EST-SNP and AFLP markers for evaluation of genetic diversity and conservation of genetic resources using wild, cultivated and elite barleys. Pl. Sci. 173: 638-649.

VEKEMANS, X. 2002. AFLP-SURV version 1.0. Distributed by the author. Laboratoire de Génétique et Ecologie Végétale, Université Libre de Bruxelles, Belgium.

VEKEMANS, X. \& O. J. HARDY. 2004. New insights from fine-scale spatial genetic structure analyses in plant populations. Molec. Ecol. 13: 921-935. PMID: 15012766. 


\section{E. N. Cerdeira et al. - Estudio de parámetros genético poblacionales en Acacia furcatispina}

VOS, P., R. HOGERS, M. BLEEKER, M. REIJANS, T. VAN DE LEE, M. HORNES, A. FRIJTERS, J. POT, J. PELEMAN, M. KUIPER \& M. ZABEAU. 1995. AFLP: a new technique for DNA fingerprinting. Nucl. Acids Res. 23: 4407-4414.

WRIGHT, S. 1951. The Genetical Structure of Populations. Ann. Eugen. 15: 323-354. http://dx.doi.org/10.1111/j.1469-1809.1949.tb02451.x

WRIGHT, S. 1978. Evolution and the Genetics of
Populations variability within and among natural populations, vol. 4. University of Chicago Press, Chicago.

YOUNG, A., T. BOYLE \& T. BROWN. 1996. The population genetic consequences of habitat fragmentation for plants. Tree 10: 413-418.

ZHIVOTOVSKY, L. A. 1999. Estimating population structure in diploids with multilocus dominant DNA markers. Molec. Ecol. 8: 907-913. 
